\title{
Effect of Benign Thyroid Disease on the Risk and Aggressiveness of Breast Cancer: An Updated Systematic Review and Meta-Analysis
}

\section{Mingyue Han}

Jilin University First Hospital https://orcid.org/0000-0003-0659-3882

\section{Xue Zhao}

Jilin University First Hospital

\section{Yao Wang}

Jilin University Second Hospital

Haiying Cui

Jilin University First Hospital

\section{Guixia Wang}

Jilin University First Hospital

Xiaokun Gang ( $\nabla$ gangxk@jlu.edu.cn )

Jilin University First Hospital

Research article

Keywords: Benign thyroid disease, Breast cancer, Aggressiveness, Meta-analysis

Posted Date: May 5th, 2021

DOl: https://doi.org/10.21203/rs.3.rs-460734/v1

License: (9) (i) This work is licensed under a Creative Commons Attribution 4.0 International License. Read Full License 


\section{Abstract}

\section{Background}

The relationship between benign thyroid disease (BTD) and breast cancer (BC) has been discussed for a long time. However, the exact connection and underlying mechanism between them remained controversial. Our meta-analysis aimed at performing a comprehensive assessment of the relationship between different types of benign thyroid disease and the risk of breast cancer, and at the same time assessing whether benign thyroid disease exerts an influence on the aggressiveness of breatst cancer.

\section{Method}

A literature search was performed using PubMed, Web of Science, MEDLINE, and Embase databases for studies published from 1982 to August 2020. All data including odds ratio (OR) and its corresponding $95 \%$ confidence intervals $(\mathrm{Cl})$ were analyzed using STATA software, version 16.0. Publication bias and quality assessment of the included studies were conducted.

\section{Result}

After strict literature search and selection, 18 articles were included in our meta-analysis. The result showed that autoimmune thyroiditis (OR 2.57, 95\% Cl 1.96-3.38), goiter (OR 2.13, 95\% Cl 1.19-3.79), and Graves' disease (OR 4.17, 95\% $\mathrm{Cl} 1.08-16.05)$ were associated with increased risk of BC. Both hypothyroidism (OR $0.82,95 \% \mathrm{Cl} 0.64-1.04)$ and hyperthyroidism (OR $1.07,95 \% \mathrm{Cl} 0.93-1.24)$ had no correlations with the risk of breast cancer. Additionally, our pooled analysis showed no significant correlation was observed between benign thyroid disease and aggressiveness of breast cancer on the whole. However, a positive relationship was found between benign thyroid disease and aggressiveness of breast cancer in the Europe subgroup (HR:2.05, 95\% Cl 1.32-3.17).

\section{Conclusion}

Among benign thyroid disease, autoimmune thyroiditis, goiter, and Graves' disease were related to the increased risk of breast cancer. As a whole, benign thyroid disease was positively associated with the risk of breast cancer. Thus, special attention should be paid to these patients during treatment and follow-up. Additionally, benign thyroid disease increases the aggressiveness of breast cancer in the European population while no significant correlation was observed in other subgroups. Thus, more large-scale cohort studies are urgently needed soon to confirm the results.

† Mingyue Han, Xue Zhao and Yao Wang should be considered shared first authors.

\section{Background}

According to the latest data from the International Agency for Research on Cancer (IARC) of the World Health Organization in 2020, the number of patients with BC increased to 2.26 million, becoming the 
most common cancer around the world ${ }^{[1,2]}$. Thus, identifying the possible risk factors and making timely prevention to reduce the incidence of $\mathrm{BC}$ is of great significance. Many risk factors such as sex, aging, estrogen, family history, gene mutations, and unhealthy lifestyle have been proven to increase the possibility of developing $\mathrm{BC}^{[3]}$. Since both thyroid and breast are regulated by the hypothalamus-pituitarygland axis, there are likely some internal relationships and mutual influences between BC and BTD.

It was Schottenfeld et al ${ }^{[4]}$ who first proposed the connection between BTD and BC. Although it failed to prove the relationship between them, it provided new insights for later researchers. So far, many scholars have investigated the relationship between BTD and BC. Some studies have shown that BTD increased the risk of $B C^{[5-15]}$, while some studies have shown that $B T D$ decreased the risk of $B C^{[16,17]}$. Besides, some studies found no connection between $B T D$ and the risk of $B C^{[15,18-22]}$. Thus, the relationship between BTD and BC remains controversial. Further research is needed on whether BTD can increase the risk of $\mathrm{BC}$.

The previous meta-analysis by Hardefeldt et al confirmed autoimmune thyroiditis (AITD), goiter, and antithyroid antibody were positively associated with the risk of BC was published in $2012^{[23]}$. However, the influence of GD, hypothyroidism, and hyperthyroidism on the risk of BC hasn't been illuminated yet, and more valuable studies on BTD and the risk of BC have been published later. Especially in recent years, many scholars focus on how BTD exerts influences on BC. Considering the ambiguous relationship, an updated meta-analysis is indispensable to identify the exact relationship between BTD and the risk of BC. Moreover, the relationship between different types of BTD and the aggressiveness of BC is unknown.

Therefore, the current update meta-analysis aimed to systematically review and compare the effects of different types of BTD on the risk of BC based on the available evidence and to identify whether there is a relation between the existence of BTD and aggressiveness of BC.

\section{Methods}

\section{Search strategy}

A systematic and comprehensive search for relevant literature was performed using PubMed, Web of Science, MEDLINE, and Embase databases up to date to August 2020. Relevant studies from databases were selected using the following keywords:" benign thyroid disease" or" autoimmune thyroiditis" or" goiter" or" hyperthyroidism" or" hypothyroidism" or" graves" AND" breast disease" or "breast neoplasms" or "mammary cancer" AND "risk" or "incidence". We assessed the title, abstract, and full text of each article for inclusion. Moreover, we checked the reference lists of the retrieved articles to identify any additional full-text articles (Figure 1). EndNote X9 was used to manage all potentially relevant articles.

\section{Study selection}

Studies should meet the inclusion criteria as follows: (1) included patients should be older than 18 years old when first diagnosed with BTD. (2) the endpoint of included patients was the diagnosis of BC and no 
antitumor therapy was given. (3) the diagnosis criteria of BTD were shown in Additional file 1

(supplement figure 1). (4) the diagnosis of BC was based on standard histopathology ${ }^{[24]}$. (5) an internal control group was available to calculate the OR. (6) we only included studies published in English in our paper, in case of missing information, we also reviewed the abstracts of non-English papers.

We excluded articles based on the following criteria: (1) patients with a family history of BC. (2) BTD patients with medication history that affect hormone levels. (3) the populations and the databases of the articles were duplicated from other published articles. (4) unable to obtain full-text or insufficient information was provided for quality assessment of the literature. (5) reviews, case reports, conference abstracts, letters, or meta-analyses.

\section{Data extraction and quality assessment}

Data from studies were separately extracted by two reviewers based on the inclusion criteria and exclusion criteria as mentioned above, discussion and consensus were achieved by a third inspector when disagreement happened. The following items were obtained from each study included in this metaanalysis: first author, year of publication, region, study design, numbers of benign thyroid diseases and breast cancer, mean age, and type of benign thyroid diseases. The quality of cohort studies was assessed by the Newcastle-Ottawa Scale (NOS) ${ }^{[25]}$ from three categories: (1)selection of research object ranged from 0 to 4 points. (2) the baseline comparability ranged from 0 to 2 points. (3) clinical outcome ranged from 0 to 3 points. The NOS score is greater than 6 was considered as high-quality literature. A score of 6 is considered to be of medium quality, while a score of less than 6 is considered to be of low quality. The quality assessment of the articles included in this meta-analysis was shown in Additional file 1

(supplement figure 2).

\section{Statistical analysis}

All data analysis was performed using STATA software, version 16.0. A pooled odds ratio (OR) and their accompanying $95 \%$ confidence intervals (Cls) were calculated for the impact of different kinds of BTD on the probability of developing $B C$ through a random-effects model. Cochran's $Q$ statistic with a $p$ value $<0.1$ was used to assess heterogeneity which indicates significant heterogeneity. When $\mathrm{I}^{2}>50 \%(\mathrm{p} \otimes 0.05)$, the study was considered heterogeneous, so a random effect model was used. On the contrary, when $1^{2} \rrbracket 50 \%$, it indicates low or moderate heterogeneity, and a fixed-effect model was used for assessment ${ }^{[26,27]}$. Data were presented in the form of forest plots. When $P$ value $<0.05$, it was considered statistically significant.

\section{Result}

\section{Search results and study characteristics}

The procedure of literature selection is based on the PRISMA statement ${ }^{[28]}$ described in Figure 1. In total, 973 records were identified through different databases, of which 7 records were identified after reviewing the reference lists of the retrieved articles. After duplicate studies $(n=289)$ were ruled out, 691 remaining 
studies have glanced over. 656 studies were excluded after screening the title and abstract. After reading the full-text of the remaining 35 articles carefully, 17 studies were excluded ( 9 articles were excluded after full-text reviewed, 4 articles were excluded without comparators included, 2 articles without suitable outcomes, and 2 articles were ruled out for unable to get full-text). Thus, 18 reports ${ }^{[5-22]}$ published between 1982 to 2020 were included in this meta-analysis.

A total of 422,384 patients were included in our study. Details about the basic characteristics of the included studies in this systematic review are summarized in Table 1. Of the included studies, 6 were from the USA, 2 were conducted in Greece, and 1 each was from Germany, China, Sweden, Brazil, Czech, Turkey, Italy, Poland, Denmark, and the UK, respectively. The countries were further divided into 2 groups to investigated the region characteristic of BTD on the risk of BC. Greece, Germany, Sweden, Czech, Italy, Poland, Denmark, and the UK belong to the Europe group, while the USA, China, Brazil, and Turkey belong to the Non-Europe group. The studies varied in sample size from 9 to 139,124. According to the NOS assessment system $^{[25]}$, all of the included articles were considered to be high-quality (Additional file 1:

\section{Supplement figure 2).}

\section{BTD increases the risk of BC}

As shown in Figure 2a, a total of 15 studies were included in the present meta-analysis. Our pooled analysis of these reports showed that a higher risk of developing BC for people with BTD (OR:1.27, $\left.95 \% \mathrm{Cl}: 1.09-1.48, \mathrm{I}^{2}=80.5 \%, \mathrm{n}=15\right)$. After stratifying by different kinds of BTD, we found that autoimmune thyroiditis (AITD) ${ }^{[5,8-11]}\left(\mathrm{OR}: 2.56,95 \% \mathrm{Cl}: 1.95-3.37, \mathrm{I}^{2}=0.0 \%, \mathrm{n}=5\right)$, and goiter ${ }^{[5,9,11,12,15]}(\mathrm{OR}: 2.13$, $\left.95 \% \mathrm{Cl}: 1.19-3.79, \mathrm{I}^{2}=80.6 \%, \mathrm{n}=5\right)$ were positively associated with the risk of $\mathrm{BC}$. However, both hypothyroidism $^{[5,6,16,19-22]}$ (OR:0.82, 95\%Cl:0.64-1.04, $\left.\mathrm{I}^{2}=85.0 \%, \mathrm{n}=7\right)$ and hyperthyroidism ${ }^{[5-7,9,11,15,18-}$ 22] (OR:1.07, 95\% Cl:0.93-1.24, $\mathrm{I}^{2}=24.9 \%, \mathrm{n}=11$ ) had no correlations with $\mathrm{BC}$ risk. Considering the varied causes of hyperthyroidism, GD is the most common cause ${ }^{[29,30]}$. Hyperthyroid patients with positive TRAb were therefore singled out as GD group to further investigated the underlying relationship between them. Our result showed a positive relationship between $\mathrm{GD}^{[7,11]}$ (OR:4.17, 95\% Cl:1.08-16.05, $\mathrm{I}^{2}=0.0 \%$, $\mathrm{n}=2$ ) and $\mathrm{BC}$ risk (Figure $\mathbf{2 b}$ ). Thus, GD increased the risk of BC was newly proposed in the present study.

To further explore the heterogeneity between the included studies, subgroup analysis was applied by different regions (Europe and Non-Europe) (Figure 2c). BTD was positively associated with the risk of BC both in Europe (OR:1.31, 95\%Cl:1.03-1.65, $\mathrm{I}^{2}=73.3 \%$ ) and Non-Europe (OR:1.27, 95\%Cl:1.01-1.61, $\mathrm{I}^{2}=84.4 \%$ ) subgroup. However, after stratification by different regions, the heterogeneity between the included studies did not show any decrease. It indicated the region characteristic did not exist for the including studies.

\section{BTD and aggressiveness of BC}

In addition to discuss the relationship between BTD and the risk of BC, we further investigated whether the existence of thyroid dysfunction exerted an influence on the aggressiveness of $\mathrm{BC}^{[31]}$. A total of 4 
pieces of research ${ }^{[13,14,16,17]}$ were included eventually. Subgroup analysis was conducted on different aggressiveness markers of $\mathrm{BC}$, grade $\otimes$ and lymph gland metastases subgroups were excluded for only one article available. Our pooled result demonstrated no relationship between BTD and grade $\nabla$ subgroup (HR:0.77, 95\%Cl:0.13-4.58, $\mathrm{I}^{2}=85.9 \%, \mathrm{n}=2$ ), tumor>20mm subgroup (HR:0.87, 95\%Cl:0.18-4.13, $\mathrm{I}^{2}=89.7 \%$, $n=2$ ), estrogen receptor-negative subgroup (HR:1.03, 95\% Cl:0.80-1.32, $\mathrm{I}^{2}=77.2 \%, \mathrm{n}=4$ ) and progesterone receptor-negative subgroup (HR:1.19, 95\%Cl:0.83-1.71, $\mathrm{I}^{2}=82.8 \%, \mathrm{n}=3$ ). The result is shown in Figure 3a. 2 studies $^{[13,17]}$ cohered with our pooled result that there is no significant relation between BTD and the aggressiveness of BC. To future investigated whether there was a difference among different kinds of BTD and aggressiveness of BC, a subgroup analysis was conducted by different kinds of BTD. After retrieving related literature comprehensively, the existing articles mainly focused on hyperthyroidism and hypothyroidism. The present synthesis analysis didn't find a relationship in hyperthyroidism (HR:1.28, 95\%Cl:0.92-1.80, $\mathrm{I}^{2}=83.9 \%, \mathrm{n}=4$ ) and hypothyroidism (HR:0.99, 95\%Cl:0.88-1.10, $\mathrm{I}^{2}=38.6 \%, \mathrm{n}=2$ ) subgroup (Figure 3b).

Considering the high heterogeneity of the included studies, we then further detected whether a difference has existed between different regions (Europe and Non-Europe). After stratification by region, the heterogeneity between the included studies did not show any decrease. However, a positive relationship was observed in Europe subgroup (HR:2.05, 95\% Cl:1.32-3.17, $\mathrm{I}^{2}=86.4 \%, \mathrm{n}=2$ ) (Figure 3c).

\section{Sensitivity analysis and publication bias}

The publication bias detection of the literature included was analyzed using the Harbord test ${ }^{[32]}$ (Figure 4). Figure 4a shows no publication bias for the relationship between autoimmune thyroiditis and $B C$ risk $(p=0.857)$. Similarly, no publication bias was observed in hypothyroidism $(p=0.287)$ and hyperthyroidism $(p=0.754)$ subgroups. However, publication bias existed in the goiter subgroup with a $p$-value of 0.019 . Sensitivity analysis ${ }^{[33]}$ was used to verify the reliability of the result of meta-analysis in the goiter subgroup. After removing the study reported by Bach et al ${ }^{[5]}$, publication bias is inexistent with a $\mathrm{p}$-value of 0.949 . The other sensitivity analysis results were consistent with the primary analysis.

\section{Discussion}

\section{Relationship between BTD and the risk of BC}

In the past few decades, there has been much literature focusing on the relationship between BTD and BC. The previous meta-analysis published in 2012 by Hardefeldt et al ${ }^{[23]}$ found AITD, goiter, and antithyroid antibodies were positively associated with the risk of BC. However, more research was published in recent years, which are precisely the largest prospective studies involving more than 400,000 patients with high qualities according to the NOS. And the relationship between BTD and BC becomes controversial. Thus, we did the updated analysis based on the available studies. Our meta-analysis concentrated on the specific types of chronic BTD, which are characterized by a longer course of the disease. Some thyroid diseases, such as acute or subacute thyroiditis were excluded and 18 studies were 
included in the present study finally. Based on all the data from the 18 articles, ATID and goiter increased the risk of $B C$ was consistent with previous studies. Besides, we found GD was related to an increased risk of BC in this meta-analysis. Subgroup analyses were further performed by region (Europe and NonEurope) and a relationship existed both in Europe and Non-Europe subgroup. After stratification by region, the heterogeneity between included studies did not show any decrease. Thus, region characteristics didn't exist in the existing literature.

\section{Underling mechanisms between thyroid autoantibody and the development of BC}

A significant feature of AITD is the existence of autoantibody, including TPO-Ab, TgAb, and microsomal. However, the diagnosis of AITD not only relies on the presence of autoantibodies but also needs evidence of thyroid dysfunction or histological confirmation ${ }^{[34]}$. It was undeniable that TPO-Ab plays an important role in the process of developing AITD. Based on the existing evidence, TPO-Ab may be a protective factor for $B C$ and a higher TPO-Ab level was associated with a lower risk of $B C^{[35,36]}$. Higher TPO-Ab level at baseline, correspond with autoimmune thyroiditis. What happens next is the development of hypothyroid with a low level of thyroid hormone. It will eventually have a protective effect on developing BC. Although

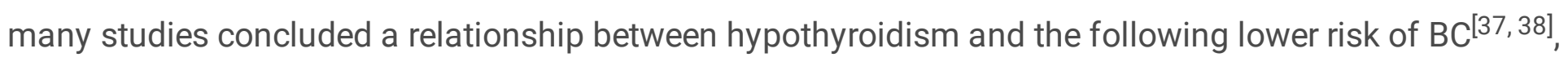
our synthesized analysis didn't reach statistically significant. This result was similar to the founding of Wang et al ${ }^{[39]}$. Prospective research also found women with high levels of TPO-Ab were at a lower risk of being diagnosed with invasive $\mathrm{BC}^{[35]}$. What can't be overlooked is that there had also been numerous cross-sectional studies ${ }^{[10]}$ that demonstrated a positive association between the level of TPO-Ab and BC, one possible explanation is that we never know whether $B C$ itself stimulated the elevating of TPO-Ab and it was the main drawback of cross-sectional studies. Cohort studies based on large numbers of the population were indispensable to draw a firm conclusion.

\section{Underlying mechanisms between hyperthyroidism and the development of BC}

The relationship between hyperthyroidism and the risk of $\mathrm{BC}$ was not concluded in the present study. After comparing with previous studies which focus on hyperthyroidism and the risk of BC roundly, we found that the studies which were added in the present study were based on a large population-based dataset and it can minimize the selection bias. However, the firm conclusions between them still can't be drawn based on existing researches. Although our pooled analysis didn't reach a statistical correlation, it was undeniable that there is a link between hyperthyroidism and the risk of BC. Many studies concluded that high pre-diagnostic fT4 level was positively associated with a high risk of $\mathrm{BC}^{[35,36,40]}$. Besides, 2 prospective cohort studies ${ }^{[13,41]}$ confirmed that hyperthyroidism increased the risk of $B C$, but they were excluded from this study for only IRR or HIR attainable. The underlying mechanisms between hyperthyroidism and BC had been studied for a long time. Several hypotheses have been suggested. It was widely accepted that sodium iodide symporter (NIS) existed both in thyroid and BC tissue and an

increased expression of the NIS in BC tissue was already demonstrated ${ }^{[42,43]}$. NIS participated in the absorption and oxidation of iodine and play a role in the development of BC. A study by Dong et al[44] 
hypothesized that incorrect positioning of NIS protein may lead to abnormal NIS expression. It will soon result in iodine deficiency, which can stimulate the secretion of gonadotropin. The over-production of gonadotropin led to high estrogen status, and such changes in endocrine status may increase the risk of $\mathrm{BC}$ and thyroid diseases. The interaction between the thyroid and mammary glands mainly involves the triiodothyronine (T3) and thyroxine (T4) pathways, and then in an estrogen-like manner to activated the thyroid hormone receptors and induced differentiation and lobular growth of the mammary gland ${ }^{[45,46]}$. Besides, overweight or obese $\left(\mathrm{BMl}>25 \mathrm{~kg} / \mathrm{m}^{2}\right)$ women with high $\mathrm{fT} 4$ were more likely to develop BC than normal-weight women, for estrogen levels are higher in obese compared to normal-weight women ${ }^{[36]}$. Thyroid hormones can enhance the effects of estrogens on BC proliferation and estrogens may act on the same receptors as thyroid hormones ${ }^{[47]}$. Interestingly, a study by Jonklaas et al ${ }^{[48]}$ found malignancy was associated with the occurrence of hyperthyroidism. As an endocrine gland, the thyroid gland had an abundant blood supply and therefore can be targeted for metastases from several non-endocrine cancers. It led to damage or destruction of thyroid tissue, started with hyperthyroidism, and then turned to hypothyroidism in the end. Compared with the previous meta-analysis, we added the results between GD and the risk of $\mathrm{BC}$. GD was considered positively associated with the risk of $\mathrm{BC}$ in the present metaanalysis. GD is the most common cause of hyperthyroidism. As we have already known, thyroidstimulating antibodies (TSAb) are the primary cause of Graves' hyperthyroidism. TSH stimulates the growth, differentiation, and function of the thyroid cells via TSHR and is a target for TSAb in the development of GD. At the same time, the expression of TSHR was found common in BC, especially with a higher prevalence in low-grade breast cancer ${ }^{[49]}$. Davies et al ${ }^{[50]}$ found TSH receptors are abundant in the fatty tissue of the mammary gland and it explained the interaction between the thyroid gland and breast tissue to some extent. Several relevant studies had already demonstrated GD increased the risk of $\mathrm{BC}$ and this conclusion was consistent with our meta-analysis. However, the sample size of included researches is small, more studies based on a large sample were needed to draw a more convincing result. In brief, the result of the present study was the most convincing for the most comprehensive literature included.

\section{BTD and aggressiveness of BC}

The relationship between BTD and aggressiveness of BC was further investigated in this meta-analysis. Larger tumors, negative ER and PGR status, and occurrence of lymph node metastases all indicated aggressiveness. Although some studies indicated that a history of hyperthyroidism was associated with an increased risk of invasive $B C$ and hypothyroidism was related to a lower risk of BC, synthesis analysis did not reach statistical significance. The study of Tosovic et al ${ }^{[14]}$ found hyperthyroidism significantly increased the risk of developing more aggressiveness $B C$, while Cristofanilli et al ${ }^{[16]}$ showed that less aggressiveness BC among hypothyroid patients.

Due to the limited quantity and high heterogeneity of studies included in this meta-analysis, we can't draw precise conclusions. Subgroup analysis was conducted by different aggressiveness markers of BC, different kinds of BTD, and regions. However, we didn't find a valuable factor that could be used as a 
parameter to decrease the heterogeneity in any subgroup. Other parameters such as age, sex, and menopausal status at the diagnosis of $\mathrm{BC}$ are not suitable in the present study, for the existing research did not provide enough data on these factors. Among the results we obtained in the present study, we found BTD increases the risk of BC in the Europe subgroup. The possible reasons for the disparity may be different gene-gene and gene-environmental backgrounds which come from different ethnicities. Because of the high prevalence and mortality of $\mathrm{BC}$ in women, it is of great value to fully understand the risk factors and aggressiveness factors and to do primary prevention. The existing evidence gave us a direction to further conduct more prospective studies to explore the influence of BTD on the aggressiveness of $B C$ and basic research is necessary to clarify how BTD exerted influence on the aggressiveness of $\mathrm{BC}$.

\section{Limitation}

Several limitations should be acknowledged. Firstly, a large portion of the included studies in our metaanalysis is cross-sectional studies. It was hard to determine the causal relationship between BTD and BC in the cross-sectional study. Secondly, the number of included articles is limited, especially for the GD subgroup. Thus, more prospective studies were needed to further illustrate the exact relationship between BTD and the BC risk. Also, there is publication bias existed in the goiter subgroup of our meta-analysis. A possible explanation for this phenomenon is that the number of the included studies in the subgroup of our article is small.

\section{Conclusion}

Among BTD, autoimmune thyroiditis, goiter, and Graves' disease were related to the increased risk of BC. As a whole, BTD was positively associated with the risk of BC. Thus, special attention should be paid to these patients during treatment and follow-up. Additionally, BTD increases the aggressiveness of BC in the European population while no significant correlation was observed in other subgroups. Thus, more large-scale cohort studies are urgently needed soon to confirm the results.

\section{Abbreviations}

BTD benign thyroid disease

$\mathrm{BC}$ breast cancer

AITD autoimmune thyroiditis

GD Graves' Disease

NIS Sodium iodide symporter

TSAb Thyroid-stimulating antibodies 
OR Odds Ratio

Cl Confidence Intervals

HR Hazard Ratio

NOS Newcastle-Ottawa Scale

\section{Declarations}

\section{Ethics approval and consent to participate}

Not applicable

Consent for publication

Not applicable

\section{Availability of data and materials}

All data generated or analysed during this study are included in this published article [and its supplementary information files].

\section{Competing interests}

The authors declare that they have no competing interests.

\section{Funding}

This work was supported by grants (XG) from the National Natural Science Fund of China (81972372, 81802753) and Foundations of Jilin Province (20170623092TC-02, 20190201034JC, 20190303152SF, $20180414047 \mathrm{GH})$. This work was also supported by grants from Foundations of the first hospital of Jilin University (JDYY52015010).

\section{Authors' contributions}

$M H, X G$ and $G W$ designed the study. $M H, X Z$ and $Y W$ wrote the paper. $Y W$ and $H C$ selected the paper. $X Z$ and $\mathrm{HC}$ did the data extraction and analysis. All authors read and approved the final manuscript.

\section{Acknowledgements}

Not applicable

\section{References}


1. Momenimovahed Z, Salehiniya H. Epidemiological characteristics of and risk factors for breast cancer in the world. Breast Cancer. 2019;11:151-64.

2. International Agency for Research on Cancer. Available from: https://www.iarc.fr/faq/latest-globalcancer-data-2020-qa/.

3. Majeed W, et al. Breast cancer: major risk factors and recent developments in treatment. Asian Pac J Cancer Prev. 2014;15(8):3353-8.

4. Schottenfeld D. The relationship of breast cancer to thyroid disease. J Chronic Dis. 1968;21(5):30313.

5. Bach L, et al. Association between thyroid gland diseases and breast cancer: a case-control study. Breast Cancer Res Treat. 2020;182(1):207-13.

6. Weng $\mathrm{CH}$, et al. Thyroid disorders and breast cancer risk in Asian population: a nationwide population-based case-control study in Taiwan. BMJ Open. 2018;8(3):e020194.

7. Szychta P, et al. TSH receptor antibodies have predictive value for breast cancer - retrospective analysis. Thyroid Res. 2013;6(1):8.

8. Jiskra $J$, et al. Thyroid autoimmunity occurs more frequently in women with breast cancer compared to women with colorectal cancer and controls but it has no impact on relapse-free and overall survival. Oncol Rep. 2007;18(6):1603-11.

9. Turken 0 , et al. Breast cancer in association with thyroid disorders. Breast Cancer Res. 2003;5(5):R110-3.

10. Gogas J, et al. Autoimmune thyroid disease in women with breast carcinoma. Eur J Surg Oncol. 2001;27(7):626-30.

11. Giani $C$, et al. Relationship between breast cancer and thyroid disease: relevance of autoimmune thyroid disorders in breast malignancy. J Clin Endocrinol Metab. 1996;81(3):990-4.

12. Adamopoulos DA, et al. Thyroid disease in patients with benign and malignant mastopathy. Cancer. 1986;57(1):125-8.

13. Søgaard $M$, et al. Hypothyroidism and hyperthyroidism and breast cancer risk: a nationwide cohort study. Eur J Endocrinol. 2016;174(4):409-14.

14. Tosovic A, et al. T3 levels in relation to prognostic factors in breast cancer: a population-based prospective cohort study. BMC Cancer. 2014;14:536.

15. Smyth PP, et al. A direct relationship between thyroid enlargement and breast cancer. J Clin Endocrinol Metab. 1996;81(3):937-41.

16. Cristofanilli $\mathrm{M}$, et al. Thyroid hormone and breast carcinoma. Primary hypothyroidism is associated with a reduced incidence of primary breast carcinoma. Cancer. 2005;103(6):1122-8.

17. Weng $\mathrm{CH}$, et al. Breast Cancer Risk in Postmenopausal Women with Medical History of Thyroid Disorder in the Women's Health Initiative. Thyroid. 2020;30(4):519-30.

18. Freitas PA, et al. Study of the Prevalence of Autoimmune Thyroid Disease in Women with Breast Cancer. Endocr Pract. 2016;22(1):16-21. 
19. Simon MS, et al. Do thyroid disorders increase the risk of breast cancer? Cancer Epidemiol Biomarkers Prev. 2002;11(12):1574-8.

20. Talamini R, et al. Selected medical conditions and risk of breast cancer. $\mathrm{Br} \mathrm{J}$ Cancer. 1997;75(11):1699-703.

21. Brinton LA, et al. Relationship of thyroid disease and use of thyroid supplements to breast cancer risk. J Chronic Dis. 1984;37(12):877-93.

22. Kalache A, Vessey MP, McPherson K. Thyroid disease and breast cancer: findings in a large casecontrol study. Br J Surg. 1982;69(7):434-5.

23. Hardefeldt PJ, Eslick GD, Edirimanne S. Benign thyroid disease is associated with breast cancer: a meta-analysis. Breast Cancer Res Treat. 2012;133(3):1169-77.

24. Schünemann HJ, et al. Breast Cancer Screening and Diagnosis: A Synopsis of the European Breast Guidelines. Ann Intern Med. 2020;172(1):46-56.

25. Stang A. Critical evaluation of the Newcastle-Ottawa scale for the assessment of the quality of nonrandomized studies in meta-analyses. Eur J Epidemiol. 2010;25(9):603-5.

26. Higgins JP, et al. Measuring inconsistency in meta-analyses. Bmj. 2003;327(7414):557-60.

27. Huedo-Medina TB, et al., Assessing heterogeneity in meta-analysis: $Q$ statistic or $I^{2}$ index? 2006. 11(2): p. 193.

28. Moher $D$, et al. Preferred reporting items for systematic review and meta-analysis protocols (PRISMAP) 2015 statement. Syst Rev. 2015;4(1):1.

29. Davies TF, et al. Graves' disease. Nat Rev Dis Primers. 2020;6(1):52.

30. Antonelli A, et al. Graves' disease: Epidemiology, genetic and environmental risk factors and viruses. Best Pract Res Clin Endocrinol Metab. 2020;34(1):101387.

31. Anastasiadi Z, et al. Breast cancer in young women: an overview. Updates Surg. 2017;69(3):313-7.

32. Furuya-Kanamori $L$, et al. $P$ value-driven methods were underpowered to detect publication bias: analysis of Cochrane review meta-analyses. J Clin Epidemiol. 2020;118:86-92.

33. Mathur MB, VanderWeele TJ. Sensitivity analysis for publication bias in meta-analyses. J R Stat Soc Ser C Appl Stat. 2020;69(5):1091-119.

34. Ragusa F, et al. Hashimotos' thyroiditis: Epidemiology, pathogenesis, clinic and therapy. Best Pract Res Clin Endocrinol Metab. 2019;33(6):101367.

35. Brandt J, Borgquist S, Manjer J. Prospectively measured thyroid hormones and thyroid peroxidase antibodies in relation to risk of different breast cancer subgroups: a Malmo Diet and Cancer Study. Cancer Causes Control. 2015;26(8):1093-104.

36. Tosovic A, et al. Prospectively measured thyroid hormones and thyroid peroxidase antibodies in relation to breast cancer risk. Int J Cancer. 2012;131(9):2126-33.

37. Liu YC, Yeh CT, Lin KH. Molecular Functions of Thyroid Hormone Signaling in Regulation of Cancer Progression and Anti-Apoptosis. Int J Mol Sci, 2019. 20(20).

38. Hercbergs A, et al. Thyroid Hormone in the Clinic and Breast Cancer. Horm Cancer. 2018;9(3):139-43. 
39. Wang B, et al. Does hypothyroidism increase the risk of breast cancer: evidence from a metaanalysis. BMC Cancer. 2020;20(1):733.

40. Khan SR, et al. Thyroid Function and Cancer Risk: The Rotterdam Study. J Clin Endocrinol Metab. 2016;101(12):5030-6.

41. Yang $\mathrm{H}$, et al. Hyperthyroidism is associated with breast cancer risk and mammographic and genetic risk predictors. BMC Med. 2020;18(1):225.

42. Tazebay $\mathrm{UH}$, et al. The mammary gland iodide transporter is expressed during lactation and in breast cancer. Nat Med. 2000;6(8):871-8.

43. Kogai T, Taki K, Brent GA. Enhancement of sodium/iodide symporter expression in thyroid and breast cancer. Endocr Relat Cancer. 2006;13(3):797-826.

44. Dong L, et al. Review of the possible association between thyroid and breast carcinoma. World $J$ Surg Oncol. 2018;16(1):130.

45. Conde I, et al. Influence of thyroid hormone receptors on breast cancer cell proliferation. Ann Oncol. 2006;17(1):60-4.

46. González-Sancho JM, et al. Thyroid hormone receptors/THR genes in human cancer. Cancer Lett. 2003;192(2):121-32.

47. Hall LC, et al. Effects of thyroid hormones on human breast cancer cell proliferation. J Steroid Biochem Mol Biol. 2008;109(1-2):57-66.

48. Jonklaas J. Infiltration of the thyroid gland by non-thyroid malignancy: A literature review reveals this to be an unusual cause of hyperthyroidism. J Clin Transl Endocrinol. 2020;20:100221.

49. Oh HJ, et al. The relationship between expression of the sodium/iodide symporter gene and the status of hormonal receptors in human breast cancer tissue. Cancer Res Treat. 2005;37(4):247-50.

50. Davies TF. The thyrotropin receptors spread themselves around. J Clin Endocrinol Metab. 1994;79(5):1232-3.

\section{Figures}




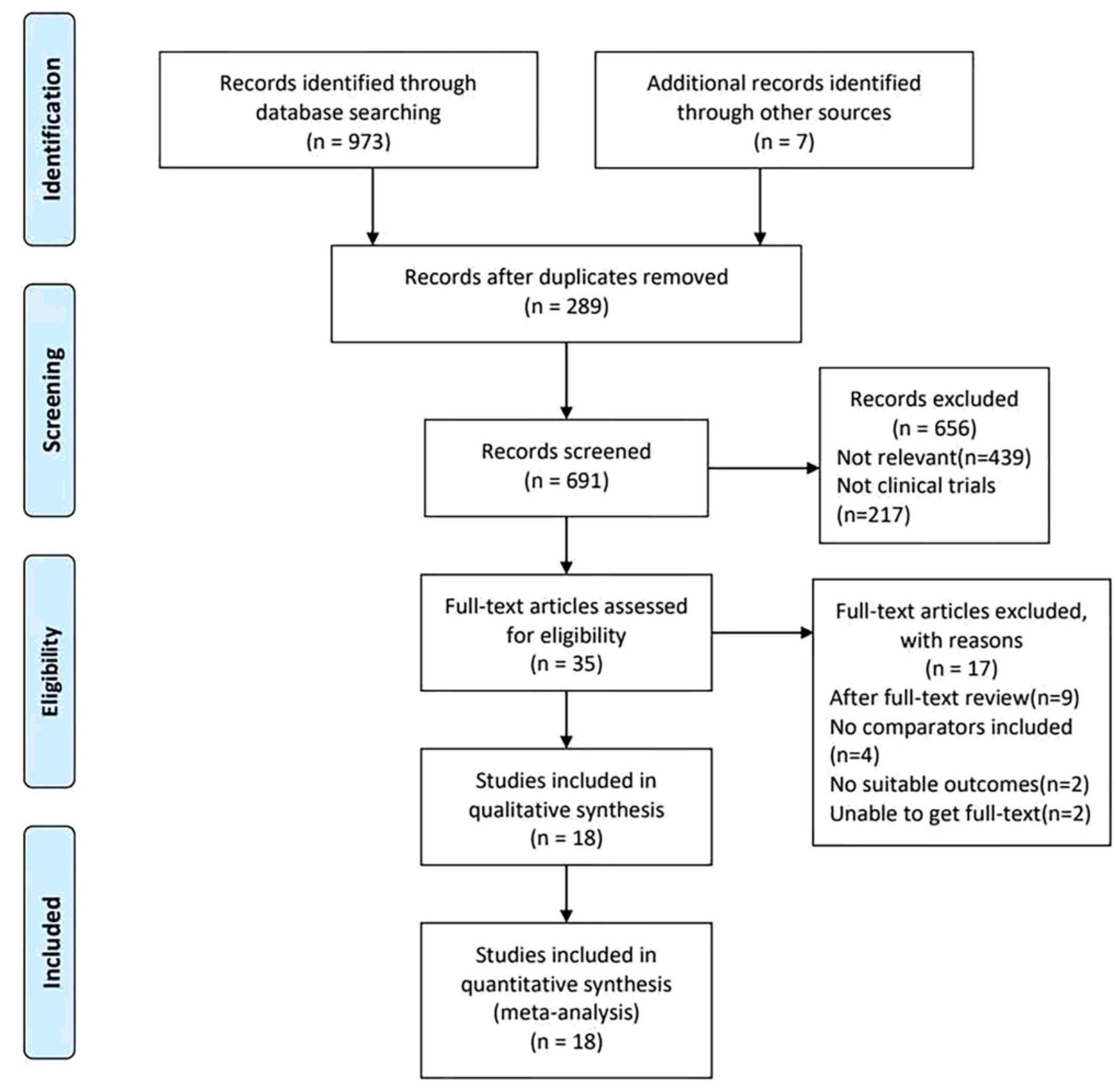

\section{Figure 1}

PRISMA Flow Diagram on the literature selection process in this meta-analysis. PRISMA Flow Diagram showing how studies were searched and screened. The flow diagram template was adapted from the 2009 PRISMA statement[28] 


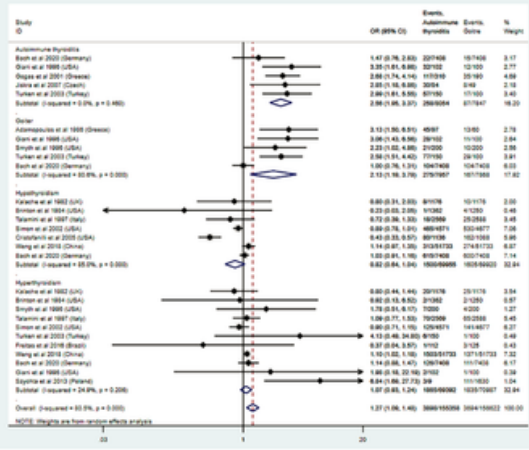

A

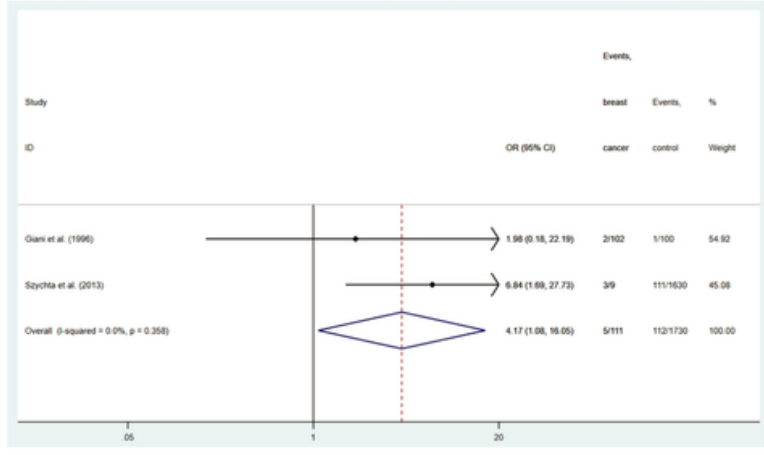

B

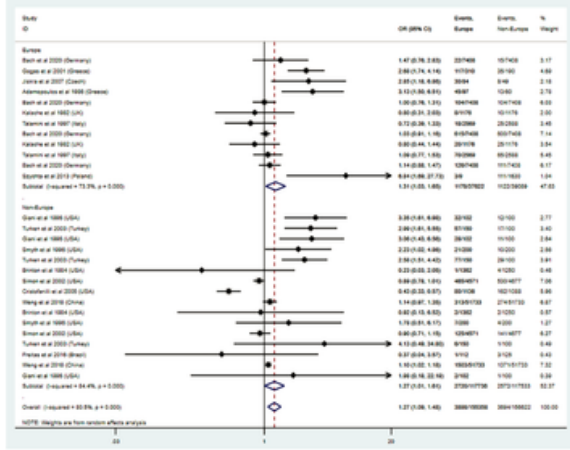

C

Figure 2

a A forest plot for assessing the association between BTD and breast cancer $(p=0.002) b$ A forest plot for assessing the association between GD and breast cancer $(p=0.002) c$ subgroup analysis on the BTD and $\mathrm{BC}$ risk.

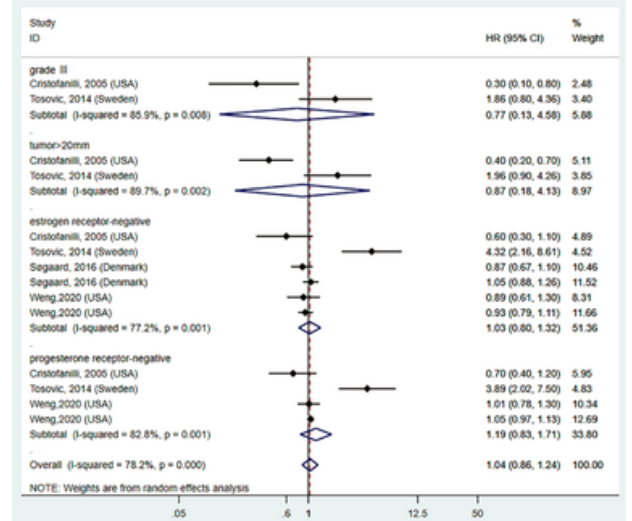

A

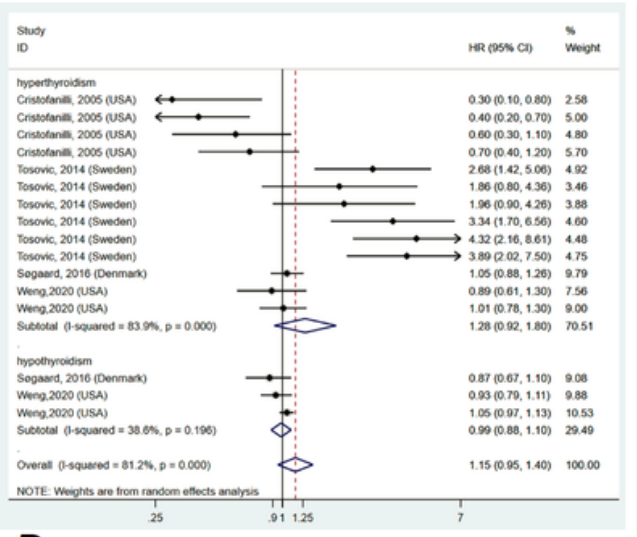

B

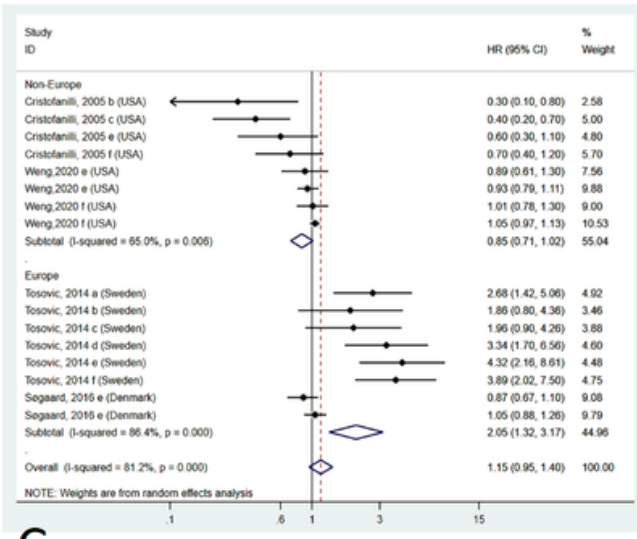

C

\section{Figure 3}

a BTD and aggressiveness of breast cancer. b subgroup analysis on the BTD and aggressiveness of BC. c subgroup analysis on the BTD and aggressiveness of $B C . a=$ grade $\nabla, b=$ grade $\nabla, c=$ tumor $>20 \mathrm{~mm}, d=$ lymph gland metastases, $e=e s t r o g e n$ receptor negative, $f=$ progesterone receptor negative 

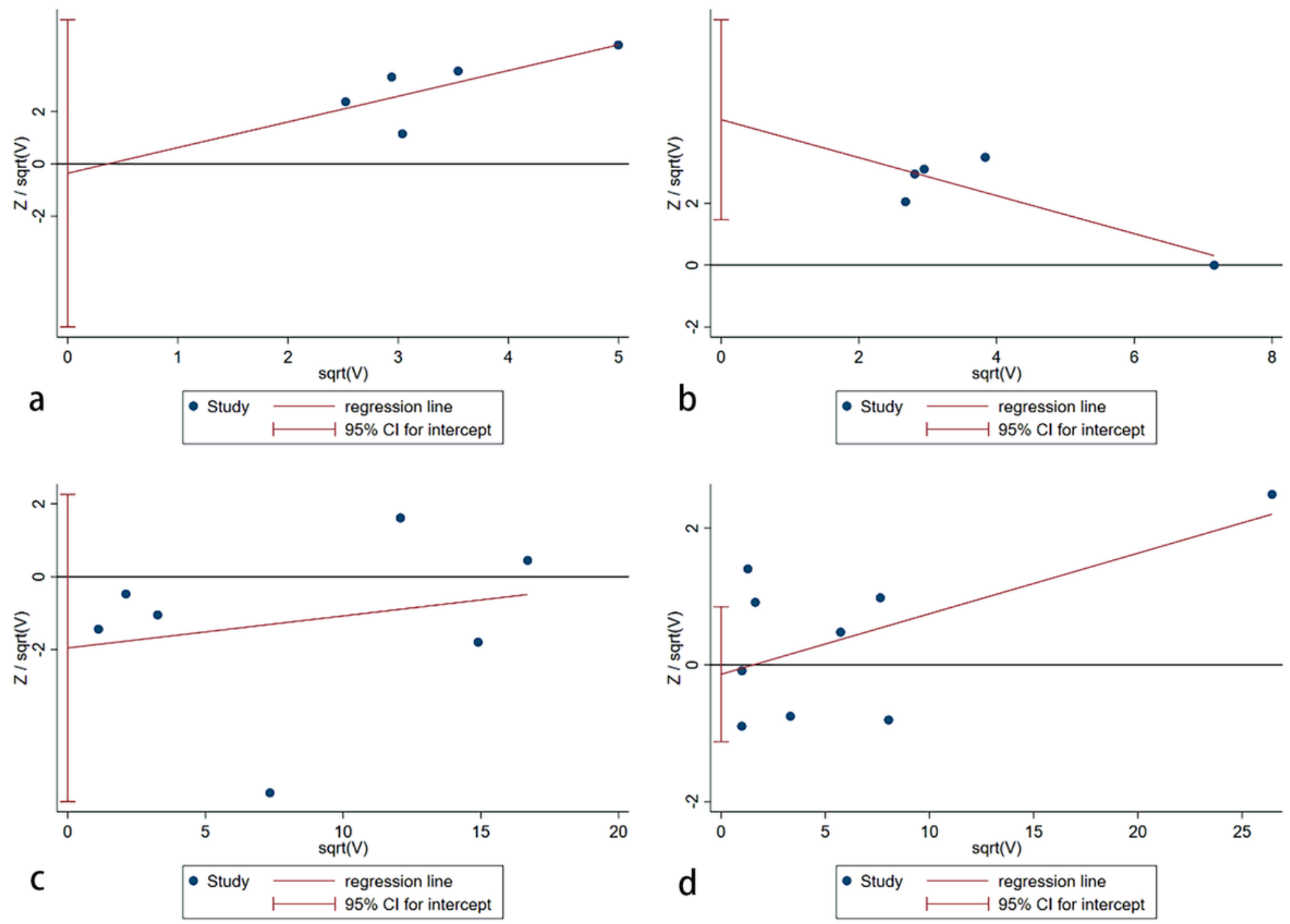

Figure 4

Publication bias assessment. a autoimmune thyroiditis; $b$ goiter; $c$ hypothyroidism; $d$ hyperthyroidism (no publication bias was found in autoimmune thyroiditis $(p=0.857)$, hypothyroidism $(p=0.287)$ and hyperthyroidism ( $p=0.754)$ subgroups. However, publication bias existed in the goiter subgroup with a $p$ value of 0.019 ) Additional file 1 (.xIsx) (Including supplement figure 1 Diagnostic criteria of benign thyroid diseases; Supplement figure 2 Quality assessment of the included studies using Newcastle-Ottawa Quality Assessment Scale)

\section{Supplementary Files}

This is a list of supplementary files associated with this preprint. Click to download.

- Additionalfile1.xlsx 